\title{
Application of SWOT-CLPV Model in China's Hierarchical Medical Mode
}

\author{
Xingyu Yang*1,a, Tianlong Shan ${ }^{2, b}$, Peiyuan Sun ${ }^{3, c}$ \\ ${ }^{1}$ School of Management engineering, Qingdao University of Technology, Qingdao, China \\ ${ }^{2}$ School of Management engineering, Qingdao University of Technology, Qingdao, China \\ ${ }^{3}$ School of Management engineering, Qingdao University of Technology, Qingdao, China
}

\begin{abstract}
Medical service system is a complex system composed of different interest related factors. The core of hierarchical medical mode is to deal with different levels and types of medical institutions to ensure the coordinated operation. In view of the reform of medical and health system, combined with the current situation of China's medical system, this paper constructs the SWOT-CLPV model of hierarchical medical in China, sorts out the advantages, disadvantages, opportunities and threats of the hierarchical medical mode in China, further explores its inhibition, problem, leverage effect and vulnerability, and studies the development strategy of hierarchical medical.
\end{abstract}

\section{Introduction}

Hierarchical medical treatment first appeared in "The Key Points of Hygiene and Birth Control in 2015", which is a report made by the national health and Family Planning Commission for a series of health work in China. This report clearly points out that it is necessary to focus on the promotion of hierarchical diagnosis and treatment to promote the in-depth development of the new medical reform, so as to realize the construction of primary diagnosis, two-way referral, rapid and slow treatment, up and down linkage hierarchical diagnosis and treatment mode Hierarchical medical service model is a medical service mode composed of two levels or multi-level, which can provide vertical division of labor and cooperation. However, with the pace of market economic system reform, different levels of medical institutions gradually deviate from the development of the times in the actual operation process, leading to confusion of functions, imperfect mechanism and other problems, and finally forming structural problems such as unreasonable distribution of medical and health resources.

Generally speaking, China's hierarchical medical mode is still in the process of exploration [1]. In this study, the advantages, disadvantages, opportunities and threats faced by the development of hierarchical medical in China are comprehensively and detailed combed in combination with domestic and foreign literature and policies. Based on the relevant information, the advantages and opportunities of hierarchical medical SWOT model are constructed, and further improved on the basis of SWOT model. Finally, the construction of SWOT-CLPV model is completed, so as to seek the best strategy and provide relevant development strategy for the development of hierarchical medical in China.

\section{SWOT-CLPV model}

SWOT analysis is also called situation analysis [2]. S represents strengths, W represents weaknesses, O represents opportunities, and $\mathrm{T}$ represents threats. This method was first proposed by management professor Weihrich in San Francisco University. Through the analysis of internal strengths, weaknesses, external opportunities and threats of target objects, it is a common tool for enterprises to carry out strategic analysis.

SWOT-CLPV model [3] is based on SWOT model for more comprehensive improvement and expansion. The focus of this method is to analyze the internal strengths and weaknesses of the target object, as well as the external opportunities and challenges of the interaction between the factors of C (control), L (leverage), P (problem) and V (vulnerability). When the internal advantages and external opportunities of the research object interact with each other, the leverage effect will evolve; when the external opportunities and internal disadvantages interact, the internal weaknesses will affect the play of external opportunities, thus producing a certain degree of inhibition on the opportunities; when the external threats and internal strengths interact, the internal strengths will be weakened, leading to vulnerability When internal weaknesses and external threats interact, it shows that there are serious problems, resulting in problem SWOT-CLPV model. At this time, qualitative analysis and quantitative analysis should be carried out. The combination of the two analysis can find out the positive and negative correlation factors, and make a more comprehensive detailed strategy. 


\section{SWOT-CLPV model of hierarchical medical}

\subsection{SWOT Model of Hierarchical Medical}

The specific SWOT analysis of hierarchical medical is shown in Table I. In general, China's hierarchical medical has many advantages. Firstly, since the national health and Family Planning Commission deployed eight tasks of public hospital reform in 2014, various favorable policies have been inclined to the direction of grassroots medical care, which has played a positive role in improving the hierarchical medical system and reasonable and orderly medical order. Secondly, "Strengthening the Grassroots" is the key content of the reform of medical and health services. With the promotion of the reform of primary health care, China has also carried out a large number of community first diagnosis and hierarchical medical practice. Thirdly, hierarchical medical is no longer limited to hospitals, but gradually dispersed, increasing health accessibility and reducing the loss of patients' inconvenience. Fourthly, The mode of close distance hierarchical medical treatment is more convenient than the only large and medium-sized hospitals in a single area. Finally Timely and efficient hierarchical medical treatment can reduce the emergency needs of patients and reduce the pressure of hospital treatment and hospitalization [4]. In addition, there are many advantages.
At the same time, hierarchical medical is also faced with many external opportunities and threats, because the current family care model is the main pension mode, and family care is often unable to timely and effectively deal with sudden diseases, especially in the current accelerated aging society [5], the chronic prevalence rate is increasing, so the elderly should be the first beneficiaries of hierarchical medical. In addition, with the development of science and technology, more and more diseases can be treated with high efficiency, which greatly resolves the one-way diagnosis and treatment of certain diseases in large hospitals. It can be seen that the development prospect of hierarchical medical treatment is broad, and further planning should be carried out. At the same time, we should also pay attention to the threat faced by hierarchical medical institutions. First of all, the current situation of our country determines that the government is difficult to bear the expenses of multi-level medical institutions. Self generated income is still the main profit channel of medical institutions. However, the "higher" medical attitude and the resources of hospitals have led to the long-term maintenance of the competitive situation. Secondly, the current lack of grassroots medical personnel flow policy, many medical college graduates in the face of choice, will naturally choose high welfare, good professional environment of medical institutions, which makes it difficult for them to enter the primary medical mode, on the contrary, most of them are secondary school graduates or individual medical workers in rural clinics Low level and quality.

Table1. SWOT analysis of hierarchical medical

\begin{tabular}{|c|c|c|}
\hline \multicolumn{2}{|c|}{ SWOT } & SWOT analysis of hierarchical medical \\
\hline \multirow{7}{*}{ Strengths } & $\mathrm{S} 1$ & Hierarchical medical policy is gradually inclined to primary medical service \\
\hline & $\mathrm{S} 2$ & Strengthening the construction of primary medical service and explore grading medical treatment \\
\hline & S3 & $\begin{array}{c}\text { Enhancing the accessibility and universality of health services and reduce the loss of patients' inconvenience in } \\
\text { medical treatment }\end{array}$ \\
\hline & S4 & Reducing the indirect health expenses such as travel expenses of patients and their families \\
\hline & S5 & Reducing the pressure of large and medium-sized hospitals \\
\hline & S6 & To some extent, it eased the tense doctor-patient relationship \\
\hline & S7 & Promoting the coordinated distribution of medical and health resources \\
\hline \multirow{5}{*}{ Weaknesses, } & W1 & The level of primary medical service is relatively weak \\
\hline & W2 & There is a lack of overall planning and layout in the construction of hierarchical medical \\
\hline & W3 & Uneven distribution of medical and health resources \\
\hline & W4 & There is a lack of qualified personnel in primary medical service \\
\hline & W5 & The level of medical publicity and public support is low \\
\hline \multirow{5}{*}{ Opportunities } & $\mathrm{O} 1$ & $\begin{array}{l}\text { With the change of the traditional way of providing for the aged, the problem of population aging is aggravating. } \\
\text { The change of population structure needs to meet the reasonable medical needs of different groups }\end{array}$ \\
\hline & $\mathrm{O} 2$ & Hierarchical medical model has been recognized and respected internationally, and gradually promoted \\
\hline & $\mathrm{O} 3$ & $\begin{array}{c}\text { The reform of medical and health system has been gradually promoted: social medical policy, long-term care } \\
\text { insurance system, and the policy of combining medical care with nursing care }\end{array}$ \\
\hline & $\mathrm{O} 4$ & The concept of medicine has changed and the scope of health service has been expanded \\
\hline & O5 & The emergence of intelligent and efficient medical equipment, Internet technology coverage and development \\
\hline
\end{tabular}




\begin{tabular}{|c|c|c|}
\hline \multirow{4}{*}{ Threats } & T1 & $\begin{array}{r}\text { There is a long-term competitive situation in the medical market, and medium and large hospitals have resource } \\
\text { advantages compared with the long-term insufficient government investment in primary health care }\end{array}$ \\
\cline { 2 - 3 } & T2 & Lack of relevant policies of medical college talent circulation \\
\cline { 2 - 3 } & T3 & Legal guarantee and policy encouragement are not in place \\
\cline { 2 - 3 } & T4 & Tense doctor-patient relationship \\
\cline { 2 - 3 } & T5 & The hospital as the core of the medical model solidified, patients lack of trust in primary medical care \\
\hline
\end{tabular}

\subsection{SWOT-CLPV Analysis of Hierarchical Medical}

\subsubsection{Control analysis (C).}

Control analysis mainly analyzes which part of the disadvantage content of hierarchical medical treatment will inhibit the role of external opportunities, which content will produce the most inhibition, and which disadvantages do not need to be considered, so as to provide reliable suggestions for the internal rectification of the industry. The specific analysis is shown in Table II. Taking the interaction between $\mathrm{O} 1$ and $\mathrm{w}$ as an example, $\mathrm{O} 1$ is "the change of traditional pension mode, the problem of population aging is aggravating, the change of population structure needs to meet the reasonable medical needs of different groups", W1 (the level of primary medical service is relatively weak), W2 (lack of overall and comprehensive planning and layout of hierarchical medical facilities), W3 (uneven distribution of medical and health resources), W4 (grassroots level) However, W5 (low level of medical publicity and public support) did not inhibit $\mathrm{O} 1$. According to the analysis of the results in the table, $\mathrm{O} 3$ is most likely to be inhibited by internal disadvantages, and the inhibition is 4C. External threats have strong inhibition, the highest is $5 \mathrm{C}$, which is difficult to change by external opportunities.

\subsubsection{Leverage effect analysis (L).}

Leverage effect analysis is mainly used to analyze the combination of internal advantages and external opportunities that can produce the greatest effect, and look for the internal advantages and external opportunities that can not play a role, and find solutions. The leverage effect of hierarchical medical is shown in Table II. It can be analyzed that the most important opportunity for hierarchical medical is O3, and the leverage effect is $6 \mathrm{~L}$. The leverage effect of $\mathrm{O} 1$ is the smallest, which is only $2 \mathrm{~L}$. Among the internal advantages of hierarchical medical, S3 leverage effect is the largest, which is $5 \mathrm{~L}$, and S2 leverage effect is the smallest, which is $1 \mathrm{~L}$.

Table2. Analysis on control and leverage effect of swot-clpv model for hierarchical medical

\begin{tabular}{|c|c|c|c|c|c|c|}
\hline Type & \multicolumn{5}{|c|}{ Opportunities } & Total \\
\hline Weaknesses & O1 & O2 & O3 & O4 & O5 & \\
\hline W1 & - & - & C & - & C & 2C \\
\hline W2 & C & C & C & - & C & 4C \\
\hline W3 & - & - & - & C & C & 2C \\
\hline W4 & - & - & C & - & - & 1C \\
\hline
\end{tabular}

\begin{tabular}{|c|c|c|c|c|c|c|}
\hline W5 & - & - & $\mathrm{C}$ & $\mathrm{C}$ & - & $2 \mathrm{C}$ \\
\hline Control total & $1 \mathrm{C}$ & $1 \mathrm{C}$ & $4 \mathrm{C}$ & $2 \mathrm{C}$ & $3 \mathrm{C}$ & $11 \mathrm{C}$ \\
\hline Strengths & & & & & & \\
\hline S1 & - & - & $\mathrm{L}$ & $\mathrm{L}$ & - & $2 \mathrm{~L}$ \\
\hline S2 & - & - & - & $\mathrm{L}$ & - & $1 \mathrm{~L}$ \\
\hline S3 & $\mathrm{L}$ & $\mathrm{L}$ & $\mathrm{L}$ & $\mathrm{L}$ & $\mathrm{L}$ & $5 \mathrm{~L}$ \\
\hline S4 & $\mathrm{L}$ & $\mathrm{L}$ & $\mathrm{L}$ & - & - & $3 \mathrm{~L}$ \\
\hline S5 & - & $\mathrm{L}$ & $\mathrm{L}$ & $\mathrm{L}$ & $\mathrm{L}$ & $4 \mathrm{~L}$ \\
\hline S6 & - & - & $\mathrm{L}$ & $\mathrm{L}$ & - & $2 \mathrm{~L}$ \\
\hline S7 & - & $\mathrm{L}$ & $\mathrm{L}$ & - & $\mathrm{L}$ & $3 \mathrm{~L}$ \\
\hline $\begin{array}{c}\text { Leverage } \\
\text { total }\end{array}$ & $2 \mathrm{~L}$ & $4 \mathrm{~L}$ & $6 \mathrm{~L}$ & $5 \mathrm{~L}$ & $3 \mathrm{~L}$ & $20 \mathrm{~L}$ \\
\hline
\end{tabular}

\subsubsection{Problem analysis $(P)$}

Problem analysis mainly explores the mutual influence between internal disadvantages and external threats of hierarchical medical, trying to find out whether there is a worse effect. The problem analysis is shown in Table III. W2 is the biggest disadvantage of hierarchical medical, and the problem analysis is 5P; T1 and T5 are the biggest threats of hierarchical medical treatment, and the problem is $4 \mathrm{P}$.

\subsubsection{Vulnerability analysis (V)}

Vulnerability analysis mainly analyzes the impact degree of the first threat of hierarchical medical treatment, and what factors can or cannot play a role after being threatened, which provides relevant basis for the development of strategic deployment. According to the analysis in Table 3, T3 has the largest vulnerability, which is $6 \mathrm{~V}$, and $\mathrm{T} 4$ is the least vulnerable, which is $1 \mathrm{~V}$; while the internal advantages are S7 and S4, both of which are $4 \mathrm{~V}$, so it is difficult to produce dominant influence.

Table3. Analysis of problem and vulnerability of swot-clpv model for hierarchical medical

\begin{tabular}{|c|c|c|c|c|c|c|}
\hline Type & \multicolumn{5}{|c|}{ Threats } & Total \\
\hline Weaknesses & T1 & T2 & T3 & T4 & T5 & \\
\hline W1 & P & P & - & P & P & 4P \\
\hline W2 & P & P & P & P & P & 5P \\
\hline W3 & P & - & - & - & - & 1P \\
\hline W4 & P & P & P & - & P & $4 \mathrm{P}$ \\
\hline W5 & - & - & P & P & P & $3 \mathrm{P}$ \\
\hline $\begin{array}{c}\text { Problem } \\
\text { total }\end{array}$ & $4 \mathrm{P}$ & $3 \mathrm{P}$ & $3 \mathrm{P}$ & $3 \mathrm{P}$ & $4 \mathrm{P}$ & $20 \mathrm{P}$ \\
\hline Strengths & & & & & & \\
\hline S1 & $\mathrm{V}$ & $\mathrm{V}$ & $\mathrm{V}$ & - & - & $3 \mathrm{~V}$ \\
\hline S2 & $\mathrm{V}$ & $\mathrm{V}$ & $\mathrm{V}$ & - & - & $3 \mathrm{~V}$ \\
\hline S3 & - & - & $\mathrm{V}$ & - & - & $1 \mathrm{~V}$ \\
\hline S4 & $\mathrm{V}$ & $\mathrm{V}$ & $\mathrm{V}$ & - & $\mathrm{V}$ & $4 \mathrm{~V}$ \\
\hline S5 & $\mathrm{V}$ & - & $\mathrm{V}$ & - & $\mathrm{V}$ & $3 \mathrm{~V}$ \\
\hline S6 & - & - & - & $\mathrm{V}$ & $\mathrm{V}$ & $2 \mathrm{~V}$ \\
\hline
\end{tabular}




\begin{tabular}{|c|c|c|c|c|c|c|}
\hline S7 & $\mathrm{V}$ & $\mathrm{V}$ & $\mathrm{V}$ & - & $\mathrm{V}$ & $4 \mathrm{~V}$ \\
\hline $\begin{array}{c}\text { Vulnerability } \\
\text { total }\end{array}$ & $5 \mathrm{~V}$ & $4 \mathrm{~V}$ & $6 \mathrm{~V}$ & $1 \mathrm{~V}$ & $4 \mathrm{~V}$ & $20 \mathrm{~V}$ \\
\hline
\end{tabular}

\section{Development strategy of hierarchical medical}

\subsection{Control and WO strategy}

The changes of pension mode, population structure and international application of hierarchical medical system are all opportunities of hierarchical medical. Compared with internal disadvantages, the opportunities encountered are more restraining. However, the level of primary medical care is weak, the distribution of health resources is uneven, and the phenomenon of talent gap in hospitals can be compensated by the current opportunities. The wo strategy should mainly make up for the disadvantages of hierarchical medical treatment: (1) make good use of the relevant national policies, adhere to the uniform distribution of medical and health resources, promote the people to gradually improve the "higher" fixed concept of seeing a doctor, steadily improve the diagnosis and treatment of common diseases and frequently occurring diseases, and provide convenient, reliable and economic diagnosis and treatment for residents. (2) We should absorb the advantages of the hierarchical medical model of other countries [6], such as the regionalized Dawson model in the United Kingdom, the unified medical service for all in Brazil, and the Caesar medical service plan, so as to achieve the goal of allocating health resources reasonably on demand and ensuring the division of labor and cooperation among medical institutions at all levels.

\subsection{Leverage effect and SO strategy}

Many advantages of hierarchical medical can adapt to the changing environment, enhance the accessibility of health services, and reduce the pressure of hospitalization and treatment in some hospitals. These advantages have a strong leverage effect. However, there is little practice of hierarchical medical in China. Therefore, strategy should not only give full play to the advantages of hierarchical medical, seize the opportunities brought by the environment, but also actively integrate the internal and external advantages. (1) Grasp the opportunity of policy change and give full play to the advantages of primary medical institutions. Make full use of the characteristics of community health institutions close to the community to provide diversified and personalized services for residents. (2) To promote the reform of China's medical and health service system and make it a supplement to the health system by carrying out the hierarchical medical model and studying the cost-effectiveness of hierarchical medical.

\subsection{Problem and WT strategy}

For the industry, the coexistence of internal and external disadvantages is the most difficult dilemma, but it can not get rid of. Facing this situation, we should not only seek external help to alleviate external threats, but also examine ourselves to make up for the damage caused by internal weakness. According to the table analysis, the most common problems are T1, T5 and internal weakness W2. Therefore, the following wt strategies are recommended: (1) promote the overall layout planning of hierarchical medical care, and reduce the adverse situation of primary medical services through reasonable allocation of medical and health resources. Common diseases and frequently occurring diseases are undertaken by a large number of primary medical institutions, and comprehensive hospitals are responsible for the diagnosis and treatment of severe and difficult diseases and related scientific research. (2) Promote the publicity channels of hierarchical medical model, truly build a good grassroots medical model, make the hierarchical medical model deeply rooted in the hearts of the people, and let the masses really feel the benefits of medical treatment brought by hierarchical medical treatment.

\subsection{Vulnerability and ST strategy}

Because of the solidification of the traditional concept of medical treatment, patients are worried about the classification of medical treatment, resulting in vulnerability, so that the internal advantages of hierarchical medical care can not play an appropriate role. The root cause lies in the insufficient capacity of primary hospitals, and many advantages are limited by external disadvantages. Therefore, St strategy can be adopted: (1) promote the state to issue corresponding policies, and the development of hierarchical medical care is the part of medical treatment that is ready to be launched. The realization of national health is inseparable from the contribution of hierarchical medical care. (2) Accurately positioning the service objects of different levels of medical institutions. The division of labor between primary hospitals and other levels of hospitals should be clear [8]. So combining with the advantages of primary medical care, improving the competitiveness of primary medical services, and making full use of these advantages and characteristics, constantly striving to standardize service behavior, can achieved the goal of adjusting the flow of patients.

\section{Conclusion}

Hierarchical medical mode is a medical service mode that needs to be expanded in the current environment, especially in the scope of epidemic control. Hierarchical medical care has its own advantages and disadvantages, while facing the opportunities and threats brought by the environment. China's hierarchical medical care is still in the exploratory stage. This study combs its strengths, weaknesses, opportunities and threats, and constructs the swot-clpv model to provide strategies for the development of hierarchical medical care. 


\section{References}

1. P. An, The Dilemma of China's Hierarchical Medical Service System and Its Breakthrough-The Construction and Promotion of Medical Association.. Changchun, Jilin: Jilin University, 2017

2. Y. Shen, "Application Progress and Prospect of SWOT Analysis," in ZHISHI JINGJI, vol. 8, pp. 76, 2009.

3. M. Chen, "SWOT--CLPV Theory and its Application," in Journal of Zhejiang Business Technology Institute, vol. 4, pp. 9-13, 2005.

4. L.D Pollina, I. Guessous, V. Petoud, C. Combescure, B. Buchs, Philippe Schaller, M. Kossovsky, JM. Gaspoz, "Integrated care at home reduces unnecessary hospitalizations of community-dwelling frail older adults: a prospective controlled trial," in BMC Geriatrics, vol.17, pp. 53-62, 2017.

5. N. Yao, C. Ritchie, T. Cornwell, B. Leff, "Use of Home-Based Medical Care and Disparities," in Journal of the American Geriatrics Society, vol.66, pp.1716-1720, 2018.

6. X. Zou, Y.Yao, P. Fang, D, Xu, C. Rao, "The Establishment of the Hierarchical Medical System: Experience and Enligntenment of Literature Abroad," in Chinese Health Economics,vol.34,pp.32-36,2015.

7. Q. Fu, "Strategic Choice for Promoting Hierarchical Treatment Model," in Chinese Health Economics, vol.34, pp.28-31, 2015.

8. Y. Zhao, "Comparatively studying the construction pathway of medical classification system in China," in Chinese Health Service Management, vol. 30, pp.244-246, 250, 2013. 